\title{
PILOT PERFORMANCE AND WORKLOAD USING SIMULATED GPS TRACK ANGLE ERROR DISPLAYS
}

\author{
Charles M. Oman ${ }^{+}$, M. Stephen Huntley, Jr. ${ }^{*}$, and Scott A. Rasmussen ${ }^{+}$ \\ +Man Vehicle Laboratory, Department of Aeronautics and Astronautics \\ Massachusetts Institute of Technology, Cambridge, MA USA \\ * Cockpit Human Factors Program, DTS-45 \\ Volpe National Transportation Systems Center, Cambridge, MA
}

\begin{abstract}
The effect on simulated GPS instrument approach performance and workload resulting from the addition of Track Angle Error (TAE) information to cockpit RNAV receiver displays in explicit analog form was studied experimentally (5 display formats, 6 pilots, 20 approaches each) in a Frasca 242 light twin aircraft simulator. Inter subject differences in ability to use the displays were found, but sliding and tilting pointer TAE formats significantly improved intercept and tracking performance measures. Determination of wind correction angle was simplified. Workload scores were not significantly influenced by display format, but depended on approach geometry and phase.
\end{abstract}

Keywords: man/machine interfaces, displays, manual control, simulators, aircraft control, flight control, air traffic control, multiloop control, mental workload, global positioning systems, navigation systems.

\section{INTRODUCTION}

Satellite based navigation systems and a new generation of microprocessor based cockpit avionics are revolutionizing air traffic control world wide. In the USA, many transport and military aircraft are now equipped with Global Positioning System (GPS) based area navigation (RNAV) computers or flight management systems, which are used for supplementary enroute and oceanic navigation. Research is underway to develop differential GPS systems with the horizontal and vertical accuracy and integrity needed for precision instrument approaches so that the aging VOR and ILS navaids can be phased out. Meanwhile, the horizontal accuracy of ordinary non-differential GPS receivers $(100 \mathrm{~m})$ is sufficently good that the Federal Aviation Administration encourages their use for less demanding nonprecision approaches, employing conventional altimetry for descent. Pilots are now permitued to fly most existing non-precision approaches using GPS as the primary reference. FAA has also begun to certify new approaches specifically designed for GPS equipped aircraft. This initiative is particularly important for the general aviation (GA) community, since non-precision approaches to thousands of new airports will eventually be possible. Because GPS approach waypoints can be arbitrarily positioned, non-traditional approach geometries can be employed to improve obstacle clearance, or reduce noise and air traffic congestion. GPS RNAVs have flexible electronic displays, updatable databases, and many more operating modes than traditional VOR, DME, IIS, and ADF equipment. The new RNAVs can potentially make instrument flying both easier and safer, provided that the human factors aspects have been properly considered at the design stage.

GPS RNAVs for civil aircraft must meet minimum performance and display standards established by the FAA (TSO C-129, and RTCA/DO-208). TSOed units are now available from some manufacturers. In most GA aircraft, instruments are of traditional type, and panel space is limited. Hence, GPS receivers are typically stand alone devices which occupy a radio or instrument slot. Only a small LCD or CRT display and a limited set of control buttons and knobs are practical. Since the GPS cross track error (XTE) 
information functionally replaces that from VOR, XTE is typically converted to an analog signal, and sent to an existing course deviation indicator (CDI), usually on a VOR head or Horizontal Situation Indicator (HSI), as shown in Fig 1. Altematively, a simulated CDI needle can be displayed on the RNAV itself (Fig. 2). As with VOR driven CDIs, the pilot always flies "toward" the needle to center it, but needle sensitivity is in linear, rather than angular units, and is scheduled: $+/-5 \mathrm{~nm}$ full scale while enroute, increasing to 1 mile during initial approach, to 0.3 miles 2 miles before final approach, and returning to 1 mile if a missed approach is flown.

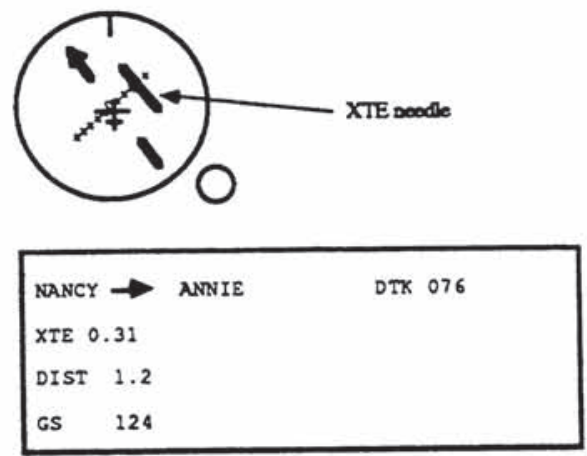

Fig. 1. (Top): Analog XTE on Horizontal Situation Indicator CDI. (Bottom): Alphameric data as they appear on nearby GPS receiver display.

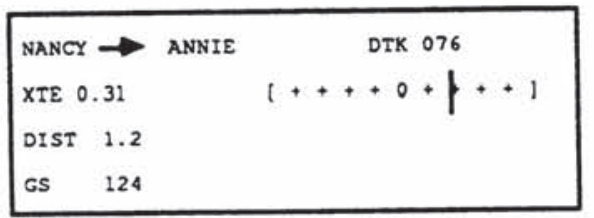

Fig. 2 Analog XTE CDI on GPS Receiver Display

Maintaining an aircraft on course centerline using XTE information is a demanding multiloop manual control task The pilot's stick position controls the third derivative of XTE, so the combined pilotaircraft system is unstable unless the pilot properly monitors and controls bank angle and heading, in addition to XTE. In a crosswind, the pilot must use a cut-and-try technique to determine the proper wind correction angle. The pilot must base his control movements in part on the rate of change of XTE to avoid large oscillations across the course centerline, particularly during the critical stages of the final approach. Judging XTE needle movement is not so easy, since the pilot also must sequentially scan attitude, heading, airspeed and altitude instruments. XTE needle sensitivity is increased on final approach to help the pilot see its movement and null small errors. However, XTE must then be even more frequently scanned, reducing the time available to perform other tasks, and increasing workload. Any side task which delays instrument scan impairs tracking performance. Inflight studies have demonstrated a direct relationship between CDI sensitivity and pilot workload, and an inverse relationship with XTE during non-precision approaches (Huntley, et al, 1991). In the parlance of manual control, the pilot's ability to create outer loop lead is determined by effective instrument scanning delay (Clement, et al, 1968). Since it is even more difficult to sense the derivative of XTE using a numeric (digital) display, TSO $\mathrm{C} 129$ requires GPS RNAVs to present XTE in analog form.

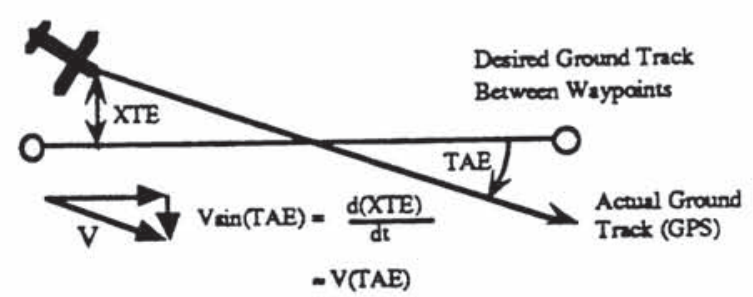

Fig. 3 The derivative of Cross Track Error (XTE) is proportional to Track Angle Error (TAE)

The capability of GPS RNAV systems to determine the direction of the aircraft's ground track with only a brief delay is potentially of importance for aircraft manual control. Since the desired heading is known, it is possible to compute "track angle error" (TAE), the difference between the desired track and the actual track. As shown in Fig. 3, TAE is mathematically proportional to the derivative of XTE, the important manual control variable. If TAE were directly displayed to the pilot, XTE could be less frequently scanned, and the pilot's performance might improve. There are several ways this could be done. One is to incorporate TAE information into inner loop attitude commands. However, many GA aircraft lack the necessary flight director equipped attitude indicator. A second possibility is to derive a TAE based "course to steer" - a heading flight director command. However, this ideally requires an additional indicator on the primary heading display not available on existing instruments. Another option is to use TAE to estimate future XTE - a "quickened XTE" display. However, information on present XTE is then lost. The fourth and perhaps simplest alternative is to present TAE information on the GPS RNAV itself in analog form. It makes sense that if TAE were explicitly displayed, pilots might learn to take advantage of it. TSOed GPS RNAVs are required to have at least a numeric display of TAE. TSO C-129 suggests that "the use of nonnumeric XTE data integrated with non-numeric TAE data into one display may provide the optimum of situation and control information for the best overall tracking performance". However, the TSO did not suggest the format of such displays, and no experimental data has yet been available as a guide to FAA or manufacturers.

The purpose of this flight simulator research project was to investigate the fourth alternative, and see how pilots used TAE information when it was presented in several different formats. Which format do pilots favor, and why? To what extent does the addition of TAE information allow pilots to quantitatively improve their approach performance, or reduce workload? If pilots must look away from their primary instruments to monitor TAE on the GPS receiver, is some of the potential advantage lost? 


\section{METHODS}

\subsection{Displays}

The TAE GPS receiver display formats evaluated were:

1) Separate TAE and XTE sliding pointer displays (2 versions). This format ${ }^{1}$, shown in Fig. 4(top) added a TAE sliding pointer display beneath a conventional, "fly to" XTE CDI. The TAE pointer was a triangle, located just beneath the XTE needle, and using the same "ten dot" (123 pixel wide) scale. When the triangle was centered, TAE was zero. Full scale TAE triangle deflection was set at \pm 90 degrees, since this is the maximum useful course intercept angle. Which way should the TAE triangle move in response to a roll command? One alternative is to have the triangle move in the same direction as the stick roll command. This is easy to remember, and has the advantage that both the needle and the triangle appear on the same side of the display when converging with course. This version was therefore referred to as "Triangle/Same". However, a concerm was that this makes the TAE triangle a "fly from" display. Since the XTE display above it is "fly to", this version apparently violates the well known human factors "command-response consistency" guideline. So we also evaluated a second version of this format, where the sign of the triangle movement was reversed. This version was referred to as "Triangle/Opposite", and is shown in Fig. 4(middle).

2) An TAE/XTE sliding/totating pointer integrated display. In this format, shown in Fig. 4(bottom), the horizontal displacement of the arrow was proportional to XTE and the tilt angle was equal to TAE. This way, dimensional correspondence was preserved for the linear and angular variables. The sign of the pointer rotation was chosen so the arrow always moved in direction of tilt. In practice, the display appeared much like a "mail slot view" of a track up moving map display, where the arrow corresponded to the desired track, and the CDI scale a a downward looking view of the aircraft's wings. If the pilot adopted this "inside out", aircraft centered frame of reference, interpretation of this display was very intuitive This format was referred to as the "Track Vector" display.

To assess the value of explicit TAE information, these three displays were experimentally compared to an "XTE only" receiver display, shown in Fig.2. All four formats required the pilot to frequently look over to the GPS receiver for XTE/TAE information, so a fifth format was also included, in which XTE was presented along with heading information on an HSI, and only alphameric information was presented on the receiver, as shown in Fig. 1. The HSI was $70 \mathrm{~cm}$ from the pilot's eye, and $9.5 \mathrm{~cm}$ beneath the attitude indicator. The GPS receiver display was created on

\footnotetext{
1 originally suggested by G. Lyddane, an FAA pilot, National Resource Specialist for Flight Management Systems, and an author of TSO C-129.
}

an high resolution LCD display, located $35 \mathrm{~cm}(27$ deg) to the right of the HSI, and which subtended approximately 10 degrees of horizontal visual angle. A consistent set of generic alphameric data was presented on all 5 displays: last and next waypoint, desired track (DTK), numeric XTE, groundspeed (GS), and distance (DIST) to waypoint. Numeric TAE was shown only on TAE displays. In all approaches, the pilot had to monitor DIST, and if a turn at the next waypoint was required, initiate a dead reckoning, standard ( $3 \mathrm{deg} / \mathrm{sec}$ ) turn at the appropriate point to intercept the next leg. Waypoints automatically sequenced when the aircraft crossed a line bisecting the angle between the inbound and outbound legs.

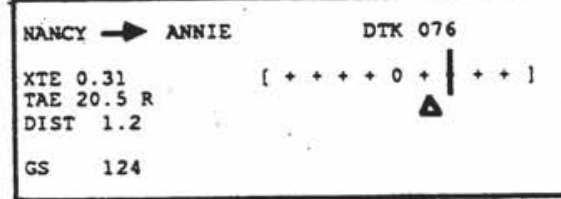

NANCY $\rightarrow$ ANNIE
XTE 0.31
TAE 20.5
DIST 1.2
GS 124

$$
\begin{aligned}
& \text { NANCY } \rightarrow \text { ANNIE } \\
& \begin{array}{l}
\text { XIE } 0.31 \\
\text { TAE } 20.52
\end{array} \\
& \text { DIST } 1.2 \\
& \text { GS } 124
\end{aligned}
$$

Fig. 4: Three GPS receiver TAE display formats studied: Top: Triangle/Same; Middle: Triangle/Opposite; Bottom: Track Vector.

\subsection{Subjects, Sessions, and Experiment Design}

Six multiengine, instrument rated pilots, were recruited locally. Total flight time averaged $1967 \mathrm{hr}$. (range 750-3387 hr.), and included an average of 73 hr. (10-213 hr.) actual instrument, $125 \mathrm{hr}$. (30-370 hr.) of simulated instrument., and $78 \mathrm{hr}$. (4-240 hr.) of time in various simulators. Multiengine experience averaged $498 \mathrm{hr}$. (22- $1500 \mathrm{hr})$. They had flown an average of $4(0-14)$ approaches and $26 \mathrm{hr} .(0-82 \mathrm{hr})$ in the past month, and an average of $6.3 \mathrm{hr} .(0-28 \mathrm{hr})$ in the week preceeding the experiment.

Each pilot flew a total of 20 approaches, four with each of the five display formats, and two different types of approach geometries. Each approach required about 15 minutes, so the experiment was conducted in two ten-approach sessions on separate days. Pilots were given a written and oral briefing on the displays and the experiment procedures. Each day, they flew the simulator and practiced with the displays until they felt familiar with them. They then flew $3-4$ complete practice approaches with the different displays to asymptote practice effects, and then flew 10 test approaches. To minimize confusion between the two triangle formats, pilots 
flew with only one of the triangle formats each day. Half the subjects flew with the triangle/same first. The presentation order of triangle displays was thus blocked, but for the three other formats was randomized and balanced within sessions.

\subsection{Aircraft Simulation and Approach Geometries}

Pilots flew a fixed base, light twin engine flight simulator (Frasca International, Inc. Urbana, IL, Model 242 ). Aircraft dynamics (furnished by Frasca) resembled a Piper Aztec. Nongaussian, patchy disturbances (Jansen, 1981) were added about the three aircraft attitude axes, independently. The ratio of roll/pitch/yaw mean disturbance was 15/5/1 times, respectively. The disturbances qualitatively resembled moderate-severe turbulence, and required the pilot to closely monitor the attitude indicator to maintain control. Additional networked computers created the GPS displays, altitude dependent wind, and collected data. Wind was always a 45 degree left or right head wind with respect to the final approach heading, but strength varied from $35 \mathrm{kts}$ at $3100 \mathrm{ft}$ above ground to $15 \mathrm{kts}$ at the surface, using a power law atomospheric model. On many legs, up to 14 degrees of heading correction was required. Pilots knew the wind direction varied, but were not told that only two relative wind directions were used.

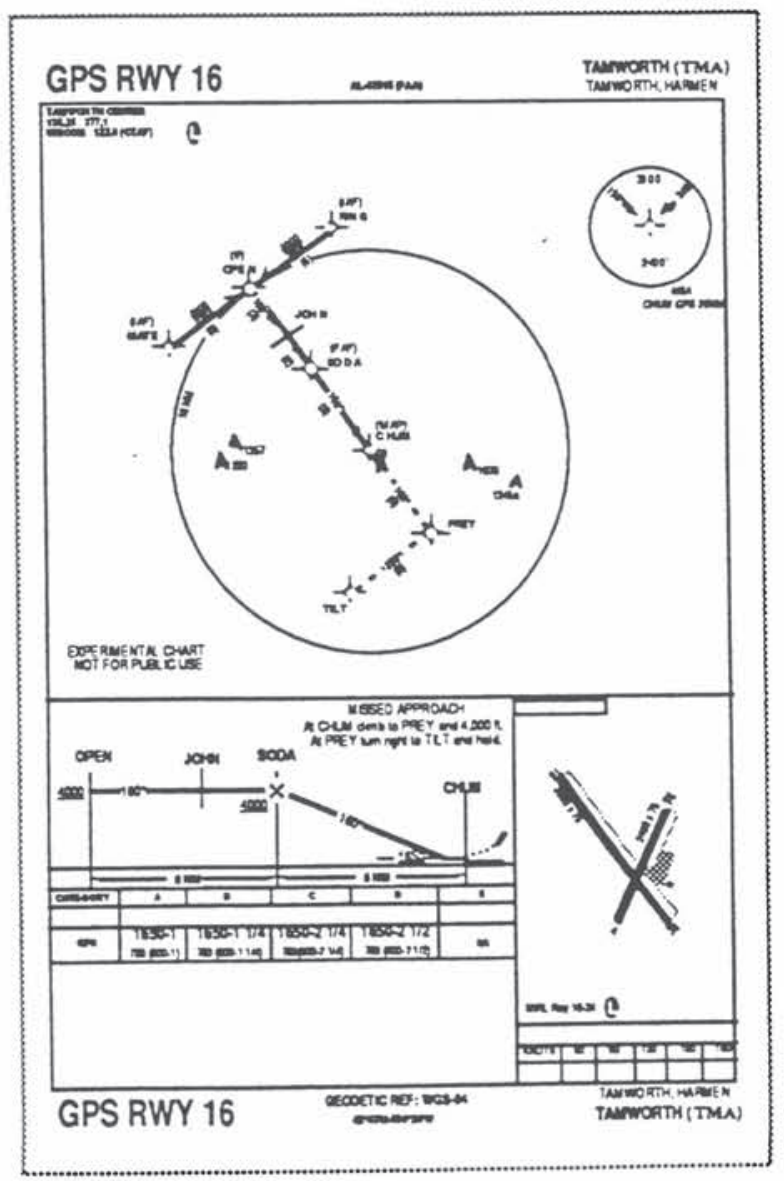

Fig. 5 Example of the NOS style GPS "T" approach plates used. Top: plan view. Bottom: elevation view, minima, and fictitious airport diagram.
Eight approach charts were employed, each with a different final approach heading and required altitudes, so that pilots could not memorize the numbers. Half the charts used a GPS " $T$ " geometry, (Fig. 5). The aircraft was initialized $0.5 \mathrm{~nm}$ upwind and abeam of the initial approach fix (IAF), located at one end of the top of the T. The pilot was required to intercept the initial approach leg, and fly five miles to an intermediate approach waypoint (IF) at the center of the " $\mathrm{T}$ ", maintaining $3100 \mathrm{ft}$. above ground level (AGL). At the IF, the pilot was instructed to turn 90 degrees, and fly five miles to the final approach fix (FAF). A waypoint 2 miles before the FAF showed where XTE CDI sensitivity changed from $\pm 1 \mathrm{~nm}$ to $\pm 0.3 \mathrm{~nm}$. The pilot was to lower the flaps and landing gear just before the FAF, and after passing it, fly five miles to the missed approach point (MAP) while descending to the $750 \mathrm{ft}$. minimum descent altitude (MDA). At the MAP, the pilot was to retract gear and flaps, and climb back to $3100 \mathrm{ft}$. AGL, flying to a first missed approach fix directly ahead five miles away, make a second 90 degree, level turn and then fly five more miles to a missed approach holding fix (MAHF). (Each chart had 2 or more IAFs, but the one used was always on the same side of the runway as the MAHF, so all turns were made in the same direction on any given run.) The turns permitted us to study performance while intercepting the subsequent leg. The initial, intermediate, and second miss legs were flown at constant altitude, and provided opportunities to measure tracking performance.

The remaining approaches used a "Crooked $T$ " geometry ${ }^{2}$ which required the pilot to also make a 45 deg. urm while initiating descent at the FAF, and then fly a two mile descending dogleg before turning back to the runway heading. There was a minimum crossing altitude at the dogleg waypoint. Since these were descending turns made with $0.3 \mathrm{~nm}$ CDI sensitivity, the Crooked $T$ approaches were expected to be much more difficult to fly.

During the approach and missed approach pilots were required to perform the usual checklist items, such as turning on and off fuel pumps, and tuning the radio to a frequency found on the chart and announcing their position. All pilots were instructed to fly the approach and the missed approach at $120 \pm 10 \mathrm{kt}$., to fly as close to course centerline as possible, to maintain altitudes within $100 \mathrm{ft}$., but never to descend below the MDA.

\subsection{Workload, Display Preference and Approach Performance Metrics}

Immediately after each approach, pilots were asked to rate the overall workload on a 10 point modified Bedford workload scale, which emphasized spare attention (Roscoe and Ellis, 1990; Huntley, et al 1993). They were asked to describe any errors made,

2 The "T" approaches resembled an approved GPS approach at Oskosh, WI. The "Crooked T" geometry was hypothetical. 
and then to rank order the 6 legs of the approach from easiest to hardest. After the second test session, a questionnaire was administered which required the pilots to subjectively rank the 5 displays using several different display preference scales. These included ease of interpretation (EOI), effect on flight path control accuracy (FPA), and overall preference (OP). In addition, each pilot was asked to indicate relative preference between individual pairs of displays on a \pm 7 point scale. The scores from these 10 pairs were summed using a tournament method, and ranked by display, to yield a second measure of overall preference, based on direct "head to head" comparisons (HTH).

XTE, TAE, altitude, airspeed, pitch and roll attitude performance parameters were sampled continuously at approx. $1 \mathrm{~Hz}$ by computer. Aircraft position data from each of the 120 approaches were rotated to a common southerly final approach heading, east/west reversed where appropriate, and ground tracks were compared by display. The combined track records were also used to retrospectively separate the approach into a series of 13 segments of varying lengths, chosen to isolate the various intercept, tracking, turning, descending and climbing phases of the approach. Mean, standard deviation, and RMS values of all six performance parameters were computed longitudinally along 13 different approach segments, and analyzed using Systat v.5.2 (Systat, Inc., Evanston, IL).

\section{RESULTS}

\subsection{Pilot Display Evaluations}

Based on pilots debriefing evaluations, TAE displays appeared to have the following advantages over the XTE only formats:

d) When intercepting a new leg, pilots could choose an appropriate intercept $\mathrm{TAE}$, and then reduce it in several steps as they approached course centerline, so as to avoid overshooting.

a) While tracking along a leg, an offset of the triangle or a tilt of the vector allowed pilots to detect and anticipate the magnitude and direction of slow changes in XTE.

b) Pilots found that they could distinguish the "diverging" and "converging" XTE/TAE pointer configurations at a glance, and react appropriately. For example, when the triangle/same pointers were on opposite sides of center, or when the track vector was tilted away from center, corrective action was immediately needed.

c) When tracking, it was possible to immediately determine the cross wind correction angle without using a cut-and-try approach. Pilots noted the heading when TAE equaled zero and many chose to set the heading indicator "bug" to this value, and simply make small left-right course corrections by flying one side of the bug. While training, pilots found it was possible to adopt a loop separation control strategy, using bank angle to control TAE and then TAE to control XTE, while effectively ignoring heading. However, closed loop control of the TAE pointer required frequent scan of the TAE display. Most pilots found this difficult or inappropriate to do in turbulence, because the attitude indicators required so much attention, and instead relied on familiar attitude and heading control strategies, using TAE to command an appropriate heading.

d) If the XTE indicator was off scale, an appropriate indication on the TAE pointer reassured the pilot that XTE would soon be on scale again.

The following TAE display deficiencies were noted:

1) Pilots said that small TAE offsets seemed more easily detected using either of the triangle displays than the vector display. The graphical display resolution was identical, but the track vector pointer showed staircasing (aliasing) when it was nearly vertical, and there was no vertical reference mark.

2) Three pilots reported confusion while interpreting the track vector display. There appeared to be two reasons for this. The first was that the format resembled a conventional CDI, not a moving map display, and there was no explicit aircraft symbol in the center. Several pilots said they had "difficulty remembering which was the airplane and which was the track". The second was that the vector would suddenly "flip" by 90 degrees during turns. Although the triangle display also flipped position during turns as the aircraft crossed the tum bisector, with the track vector display the pilot needed to mentally rotate his frame of reference when the vector flipped in order to maintain the "inside out" map interpretation. Several pilots found they could not always do this.

\subsection{Pilot Display Preferences}

Post-session questionnaire data clearly indicated a preference for the triangle/same display over either of the other two TAE displays. At the same time, the rankings underscored the relative importance to the pilots of having XTE information in the primary instrument scan area, rather than alone on the GPS receiver. Results are shown in Table 1 . A statistically significant effect of display was found for the HTH, FPA, and EOI scale rank scores from the individual subjects (Friedman rank ANOVA, $p<0.04$ ). An OP scale display effect was found at the $\mathrm{p}<0.06$ level.

On the Overall Preference (OP) and Head-To-Head (HTH) comparison scales, pilots consistently preferred the HSI display over the "triangle/same" TAE display. However, in terms of summed rank scores, the margin was slight. When asked to make head to head comparisons, half the pilots preferred the triangle/same display, two preferred the HSI display, and one judged it a tie.) Directly comparing the "triangle/same" and "triangle/opposite" versions, 4 pilots preferred the former, and only one the latter. 
All pilots always ranked one or more of the TAE display formats above the XTE only on both scales, so the consensus was clear that TAE information was subjectively useful.

\section{Table 1. Pilot Display Preference Ranks by display} using 4 different scales (see text). Rank = 1 is best.

\begin{tabular}{lcccc}
\hline Display & \multicolumn{3}{c}{ Display Preference Scales } \\
Format & OP & HTH & FPA & EOI \\
\hline ASame & 2 & 2 & 1 & 2 \\
N VOpposite & 3 & 4 & 2 & 5 \\
Vector & 4 & 3 & 4 & 3 \\
HSI & 1 & 1 & 3 & 1 \\
XTE only & 5 & 5 & 5 & 4 \\
\hline
\end{tabular}

For accurate flight path control, pilots preferred the triangle/same display, though the three subjects $(1,4$ and 5) who actually tracked most consistently (see below) ranked the HSI first in this respect. Four of the five pilots said the HSI was the easiest of the displays to interpret, though three of the four cited long-standing training and experience with the HSI format as one reason for this preference.

Four of the six pilots said they never referred to the numeric TAE information at all, since it was not obvious how to interpret the L/R TAE indication, and because the analog TAE pointer was available.

\subsection{Influences on Subjective Workload}

Average Bedford subjective workload was 3.5 out of 10 on $T$ approaches, and increased to 4.5 on Crooked T. Subject 2 had the highest average workload (6.2) and Subject 1 the lowest (3.0). ANOVA of modified Bedford Workload scores revealed significant effects between subjects $(\mathrm{F}(5,108)=29 ; \mathrm{p}<.0001)$, and $\mathrm{T}$ and Crooked $\mathrm{T}$ approach types $(F(2,108)=28.4 ; p<.0001)$. The average workload scores by display type were: triangle/same $=3.8$; triangle/opposite $=3.9$; track vector=4.0, HSI=3.9; XTE only=4.5. However, adding display to the ANOVA did not produce a significant effect. No trends were found by sequential approach or session number, suggesting training had asymptoted practice effects. Ranking workload scores within subjects did not reveal a display dependent effect. It was concluded that display effects, if they exist, must be small compared to approach geometry and inter subject effects.

The pilots ranked the approach legs in order of decreasing difficulty: 1) long/dogleg final, 2) short final, 3) intermediate leg, 4) first miss , 5) initial leg, and 6) second miss leg. Ranking was identical for both approach geometries, and the concordance of workload rankings within geometries was statistically significant (Friedman ANOVA, df $=5 ; p<0.0001$ )

\subsection{Display Effects on Track and Performance}

The reoriented aircraft ground tracks are shown superimposed in Fig. 6, staggered by the five display types. The Crooked T tracks can be distinguished by the dogleg after the FAF. Several approaches where pilots made gross errors are apparent, particularly for the XTE only display (upper right). The frequency of such errors was noted to be less with the HSI and triangle/same displays (left and third from left).

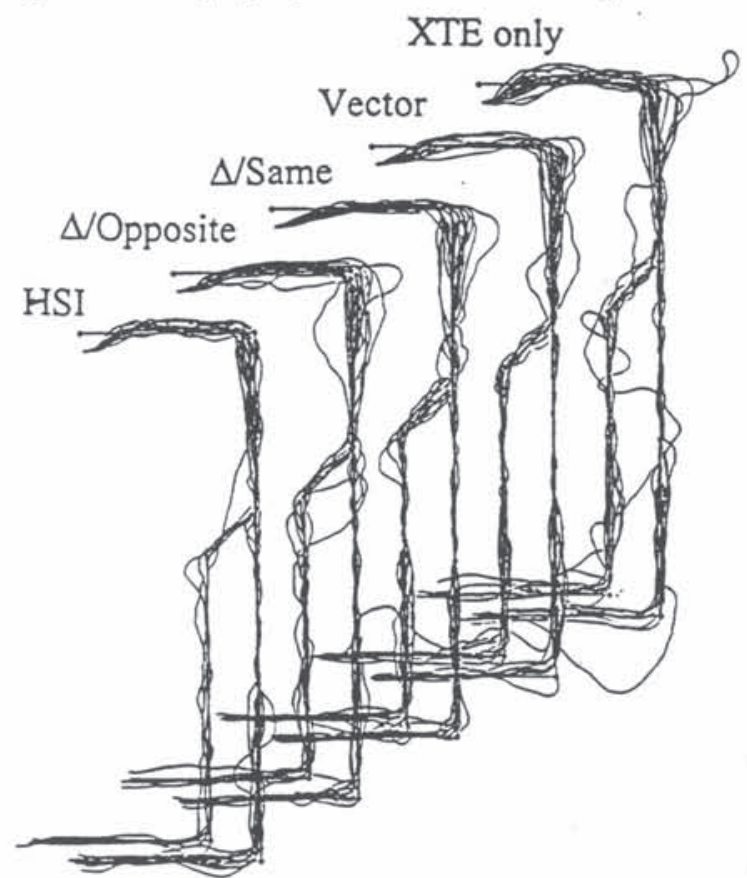

Fig. 6. Reoriented ground tracks by display type.

When the simulation began, the pilot's first task was to intercept the initial approach leg. Crosswind (always from the right in Fig. 6) tended to steepen the intercept angles of the non-TAE display approaches. Pilots flying with the triangle/same and vector displays had smaller downwind biases, as shown in Fig. 7. The difference in average tracking bias was significant by display $(F(4,90)=7.8, p<.001)$ and subject. $(F(5,90)=7.8 ; p<.001)$. However, the display effect was not significant for the second miss leg, possibly because pilots knew the wind direction by then, and the workload on this leg was lower, so pilots may have paid more attention to nulling XTE.

For the tracking portions of the initial (miles 2-4), intermediate (miles 1-3), and second missed approach (miles 1-4.5) legs, ANOVA showed significant effects of display $(F(4,329)=2.7, P<0.03)$ and subject $(F(5,329)=14.8, p<0.0001)$ in the combined data for standard deviation of XTE and also standard deviation of TAE (display: $F(4,168)=4.9, p<.001$; subject: $F(5,168)=11, p<.0001)$. This was consistent with the notion that pilots were controlling TAE. For this and other approach segments tested, a significant subject by display interaction term was found, suggesting that certain subjects learned to make better use of the TAE information than others. For most segments, the HSI or Triangle/same displays generally ranked best, followed by the track vector display. Average display effects were smaller than subject effects. The standard deviation of XTE was consistently lower on the final approach leg, as the aircraft approached the MAP. The standard 
deviation of XTE on final approach $(0.09 \mathrm{~nm})$ with the "XTE only" display was quantitatively similar to that obtained in a comparable flight study $(0.06 \mathrm{~nm})$, in a Beech 55 Baron, where XTE also was displayed on a separate CDI (Huntley, 1993).

Inter subject differences in XTE and TAE tracking performance appeared to relate in part to inner loop attitude control. Two subjects ( 2 and 6 ) consistently showed larger values of standard deviation of pitch and roll attitude, airspeed, and altitude, suggesting that their effective attitude instrument scanning delay was longer. There was no clear effect of display format in the longitudinal axis on pitch, airspeed, and altitude, but for the lateral/directional axis, ANOVA revealed a significant effect of both subject $(F(5,449)=59, p<.0001)$ and display $(F(4,449)=3.9$, $\mathrm{p}<.004)$ on the variation in roll attitude. No correlation of performance with low recency or experience was found, except for pilot 2, whose recent instrument time was only in helicopters.

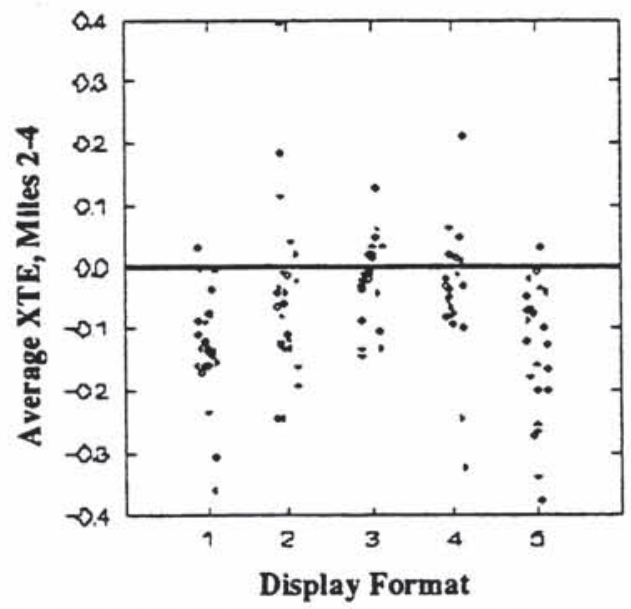

Fig. 7. Average XTE for miles 2 - 4 of Initial Approach Leg, by Display Type. Key: 1=HSI, $2=\Delta /$ opposite, $3=\Delta /$ same; $4=$ vector; $5=X T E$ only.

\section{CONCLUSIONS}

Results showed that even under turbulence conditions requiring diligent attitude instrument scan, the addition of analog TAE information to a GPS receiver XTE display significantly improved approach leg intercept and tracking performance measures, probably by allowing the pilot to predict XTE changes. Determination of wind correction angle was greatly simplified. Pilots chose not to use the numeric TAE information provided. Thus, the study quantitatively supports the FAA TSO-C129 recommendation for analog TAE displays.

Certain pilots were better able to improve their performance with TAE displays than others. Of the three TAE display formats evaluated, a sliding TAE pointer located beneath the XTE CDI, moving in the same direction as aircraft bank, produced the largest performance improvement, and was preferred by the pilots for flight path control. However, control tests with HSI displaying heading and XTE (but not TAE) on the primary instrument panel showed that the performance advantages of adding TAE to the receiver display were partly lost because of the need to widen the pilot's scan. The simultaneous display of XTE on the HSI and XTE/TAE information on the GPS receiver has not yet been tested.

The integrated XTE/TAE "track vector" display evaluated in this study had the advantage that pilots could visualize it as a track up moving map. However, it was found to be less useful for small TAE corrections because it lacked a vertical reference line. The display resembled a conventional CDI, rather than a moving map, and pilots occasionally had difficulty maintaining the map interpretation, particularly during turns when a cognitive mental rotation was required to reinterpret the display after waypoint changeover. The addition of a fixed, central aircraft reference symbol and the use of a surrounding outline box may improve this display.

Although Bedford scores were sensitive to approach geometry, no consistent effect of display format on workload was found. It is possible that our pilots adjusted their performance criteria so that workload remained approximately constant, and performance varied instead.

\section{ACKNOWLEDGMENTS}

We thank G. Lyddane, S. Robinson, D. Hannon, T. Carpenter-Smith, J. Bastow, F. Sheelen, J. Giurleo, and J. Turner, who all made important conceptual or technical contributions, and also our subjects. Protocol was approved by IRBs at MIT and DOT/Volpe. Supported by MIT CTS/DOT-Volpe Contract DTRS-57-92-C-0054 TTD\#27A.

\section{REFERENCES}

Clement, W.F., Jex, H.R., Graham, D. (1968). A Manual Control-Display Theory Applied to Instrument Landings of a Jet Transport. IEEE Trans. Man Machine Sys. 9:4 93-110

FAA (1994) Technical Standard Order C129:Airborne Supplemental Navigation Equipment Using the GPS. FAA, Washington, DC.

Huntley, M.S. Jr. (1993) Flight Technical Error for Category B Non-Precision Approaches and Missed Approaches Using Non-Differential GPS for Course Guidance. Report DOT-VNTSC-FAA-93-17

Jansen, C.J. "Non-Gaussian Atmospheric Turbulence Model for Flight Simulator Research." AJAA_L. Aircraft 19 (1981): 374-379.

Roscoe, A.H. and Ellis, G.A., (1990) A subjective rating scale for assessing pilot workload in flight. Tech. Report TR90019, RAE Famborough, UK.

RTCA Special Committee 15. (1991) Minimum Operational Performance Standards For Airbourne Supplemental Navigation Equipment Using Global Positioning System, DO-208 July, 1991, 1140 Connecticut Avenue, N.W., Washington, DC. 